\title{
Imaging the Intracranial Atherosclerotic Vessel Wall Using 7T MRI: Initial Comparison with Histopathology
}

A.G. van der Kolk, J.J.M. Zwanenburg, N.P. Denswil, A. Vink, W.G.M. Spliet, M.J.A.P. Daemen, F. Visser, D.W.J. Klomp, P.R. Luijten, and J. Hendrikse

\begin{abstract}
BACKGROUND AND PURPOSE: Several studies have attempted to characterize intracranial atherosclerotic plaques by using MR imaging sequences. However, dedicated validation of these sequences with histology has not yet been performed. The current study assessed the ability of ultra-high-resolution 7T MR imaging sequences with different image contrast weightings to image plaque components, by using histology as criterion standard.
\end{abstract}

MATERIALS AND METHODS: Five specimens of the circle of Wills were imaged at 7T with $0.11 \times 0.11 \mathrm{~mm}$ in-plane-resolution proton attenuation-, T1-, T2-, and T2*-weighted sequences (through-plane resolution, $0.11-1 \mathrm{~mm}$ ). Tissue samples from 13 fiducial-marked locations (per specimen) on MR imaging underwent histologic processing and atherosclerotic plaque classification. Reconstructed MR images were matched with histologic sections at corresponding locations.

RESULTS: Forty-four samples were available for subsequent evaluation of agreement or disagreement between plaque components and image contrast differences. Of samples, $52.3 \%(n=23)$ showed no image contrast heterogeneity; this group comprised solely no lesions or early lesions. Of samples, $25.0 \%$ ( $n=11$, mostly advanced lesions) showed good correlation between the spatial organization of MR imaging heterogeneities and plaque components. Areas of foamy macrophages were generally seen as proton attenuation-, T2-, and T2*- hypointense areas, while areas of increased collagen content showed more ambiguous signal intensities. Five samples showed image-contrast heterogeneity without corresponding plaque components on histology; 5 other samples showed contrast heterogeneity based on intima-media artifacts.

CONCLUSIONS: MR imaging at 7T has the image contrast capable of identifying both focal intracranial vessel wall thickening and distinguishing areas of different signal intensities spatially corresponding to plaque components within more advanced atherosclerotic plaques.

ABBREVIATIONS: COW = circle of Willis; $\mathrm{PD}=$ proton-attenuation

ntracranial atherosclerosis is emerging as one of the main causes of cerebral ischemic stroke and transient ischemic attack, with a high risk of recurrent ischemic events. ${ }^{1}$ In recent years, several

Received June 18, 2014; accepted after revision August 30.

From the Departments of Pathology (A.V., W.G.M.S.), Radiology (A.G.v.d.K., J.J.M.Z., F.V., D.W.J.K., P.R.L., J.H.), and Image Sciences Institute (J.J.M.Z.), University Medical Center Utrecht, Utrecht, the Netherlands; Department of Pathology (N.P.D., M.J.A.P.D.), Academic Medical Center Amsterdam, Amsterdam, the Netherlands; and Philips Healthcare (F.V.), Best, the Netherlands.

This research was performed within the framework of the Center for Translational Molecular Medicine (www.ctmm.nl), project PARISk (Plaque At RISk; grant 01C-202), and was supported by the Dutch Heart Foundation.

Please address correspondence to Anja G. van der Kolk, MD, PhD, Department of Radiology, University Medical Center Utrecht, Postbox 85500, 3508 GA, Utrecht, the Netherlands; e-mail: A.G.vanderKolk@umcutrecht.nl

Indicates article with supplemental on-line tables.

Indicates article with supplemental on-line photo.

http://dx.doi.org/10.3174/ajnr.A4178
MR imaging sequences have been developed on 3T and 7T field strengths that specifically visualize the intracranial arterial vessel wall, enabling direct assessment of intracranial atherosclerotic plaques. ${ }^{2-8}$ Similar to studies of carotid artery atherosclerosis almost a decade ago, several studies have recently attempted to characterize intracranial plaque components, such as intraplaque hemorrhage, ${ }^{9,10}$ fibrous cap, ${ }^{11}$ and lipid components, by using MR imaging. ${ }^{12,13}$

For the carotid arteries, much research has already been done validating image signal heterogeneity within the vessel wall with histology, the criterion standard. ${ }^{14-20}$ Imaging carotid artery atherosclerosis has the advantage of easy access to ex vivo atherosclerotic plaque material for validation, using carotid endarterectomy specimens. It is now possible to image calcification, fibrous cap, intraplaque hemorrhage, and lipid-rich necrotic core in the carotid artery with moderate-to-good sensitivity and specificity by 
using multicontrast MR imaging. ${ }^{20}$ Although 1 recent study showed promising preliminary results of plaque characterization by using a combined T1- and T2-weighted sequence ${ }^{21}$ compared with histology, dedicated validation with histology of intracranial vessel wall sequences with multiple image contrast weightings has not yet been performed. Therefore, whether MR imaging with multiple image contrast weightings has enough image contrast to also visualize various intracranial atherosclerotic plaque components remains a question.

Validation of MR images with histology for intracranial atherosclerosis in vivo is much more cumbersome compared with carotid plaques, because no therapies (comparable with carotid endarterectomy) exist in which intracranial atherosclerotic plaques are removed. Furthermore, intracranial arteries are smaller than carotid (or other major peripheral) arteries, ${ }^{22}$ necessitating a high spatial resolution, and therefore high SNR, for plaque visualization. Because the SNR increases approximately linearly with field strength, 7T MR imaging might provide the spatial resolution necessary to image small atherosclerotic plaques. ${ }^{22}$ Furthermore, several dedicated intracranial vessel wall sequences at 7T have already shown promising results in the visualization of vessel wall lesions in vivo.

In this feasibility study, ultra-high-resolution 7T MR imaging sequences with different image contrast weightings were developed and used in an ex vivo setting, to assess the ability (image contrast) of 7T MR imaging to image different intracranial atherosclerotic plaque components. For validation of our findings, results were compared with histology.

\section{MATERIALS AND METHODS Specimen Preparation}

Five specimens of the circle of Willis (CoW) were selected from $>100$ postmortem cases that were performed in our institution. Material was handled in a coded manner that met the criteria of the code of conduct used in the Netherlands for the responsible use of human tissue in medical research (www.federa.org/codesconduct), and institutional review board approval for this retrospective study was obtained. The primary selection criterion was the macroscopic presence of atherosclerosis as judged by an experienced pathologist (A.V.). Furthermore, care was taken that the specimens included the major arteries of the CoW; if specimens were damaged and therefore not complete (ie, histologic samples could not be taken from all 13 locations described below), they were excluded from our study, even when macroscopic atherosclerosis was present. All specimens had been stored in formaldehyde. After selection, the specimens were cleaned thoroughly with a lukewarm solution of polysorbate 20 (0.5\% Tween-20; Sigma Aldrich, St. Louis, Missouri) dissolved in distilled water to remove blood clots within the arterial vessel lumen, reducing the chance of artifacts on MR imaging. The specimens were then individually placed within a $9.5-\mathrm{cm}$ round Petri dish and embedded in a $2 \%$ agarose solution (Seakem LE Agarosel; Lonza, Rockland, Maine). During immersion in the agarose solution, care was taken to remove all air from the specimens. The embedded specimens were stored in a refrigerator at a temperature of $5^{\circ} \mathrm{C}$ for solidification until imaging could be performed.

To enable spatial correlation of the MR images with histologic sections, we used cactus spines as fiducials and placed them in the agarose gel, adjacent to 13 locations of histologic sampling. The 13 locations (Fig 1) included, when possible (no air within artery), all the major arteries of the CoW: vertebral arteries, basilar artery (different levels), posterior cerebral arteries, distal ICAs, MCAs, and anterior cerebral arteries.

\section{MR Imaging Protocol}

Imaging was performed on a 7T whole-body system (Achieva; Philips Healthcare, Best, the Netherlands) with two 16-channel dedicated surface coils (High density surface array; MR Coils BV, Drunen, the Netherlands) above and below the specimens for signal reception, ${ }^{23}$ and a volume transmit/receive coil for transmission (Quad TR; Nova Medical, Wilmington, Massachusetts). Two specimens could be imaged at one time. The specimens, within their Petri dish, were placed on top of each other in the middle of a thin plastic container, by using plastic filling above and below. The container was then filled with fluorinated lubricants (Fomblin PFPE; Solvay Solexis, Bollate, Italy) until the specimens were completely submerged, to provide susceptibility matching. The two 16-channel surface coils were placed above and below the filled box, after which the whole package was placed inside the volume transmit/receive coil.

Imaging was performed at room temperature. For imaging, sequences with 4 different contrast weightings were used, resembling previous MR imaging-histologic correlation studies in the proximal ICA. Parameters of the 4 sequences can be found in On-line Table 1. After a 3D T1-weighted turbo field echo sequence with full-specimen coverage was applied, single-section proton-attenuation (PD)-weighted spin-echo-, single-section T2-weighted TSE, and single-section T2*-weighted turbo field echo sequences with identical geometric parameters were performed for each of the 13 marked locations (resulting in 1 section per image contrast per sample location), by using the T1weighted images for planning. The PD-weighted spin-echo and T2-TSE sequences included an inversion pulse to null the signal from the agarose gel, similar to CSF suppression in a FLAIR sequence. The inversion delay of $1100 \mathrm{~ms}$ was regarded as long enough to limit the amount of T1-weighting in the vessel wall, which has a shorter $T 1$ ex vivo (approximately $300 \mathrm{~ms}$; data not shown). The total scan duration for each CoW specimen was approximately 40.5 hours. To compensate for potential frequency drifts due to the long sequence durations, we used frequency navigators. For the PD-weighted spin-echo and T2-TSE, the nonselective frequency navigators were applied just before excitation and used to adjust the system frequency accordingly. For the T2*weighted turbo field echo sequence, a navigator echo was used, as described previously. ${ }^{24}$

\section{Histologic Processing}

Histologic processing was performed by using an in-house-developed protocol. After imaging was performed, approximately 0.5 $\mathrm{cm}$-thick tissue samples were taken from the 13 marked locations of each CoW specimen. Ink markings were then used to enable correlation of histologic sections and MR images: Black ink was used to mark the sample side next to the fiducial, while blue ink was used to mark the cranial side of the sample with respect to the 

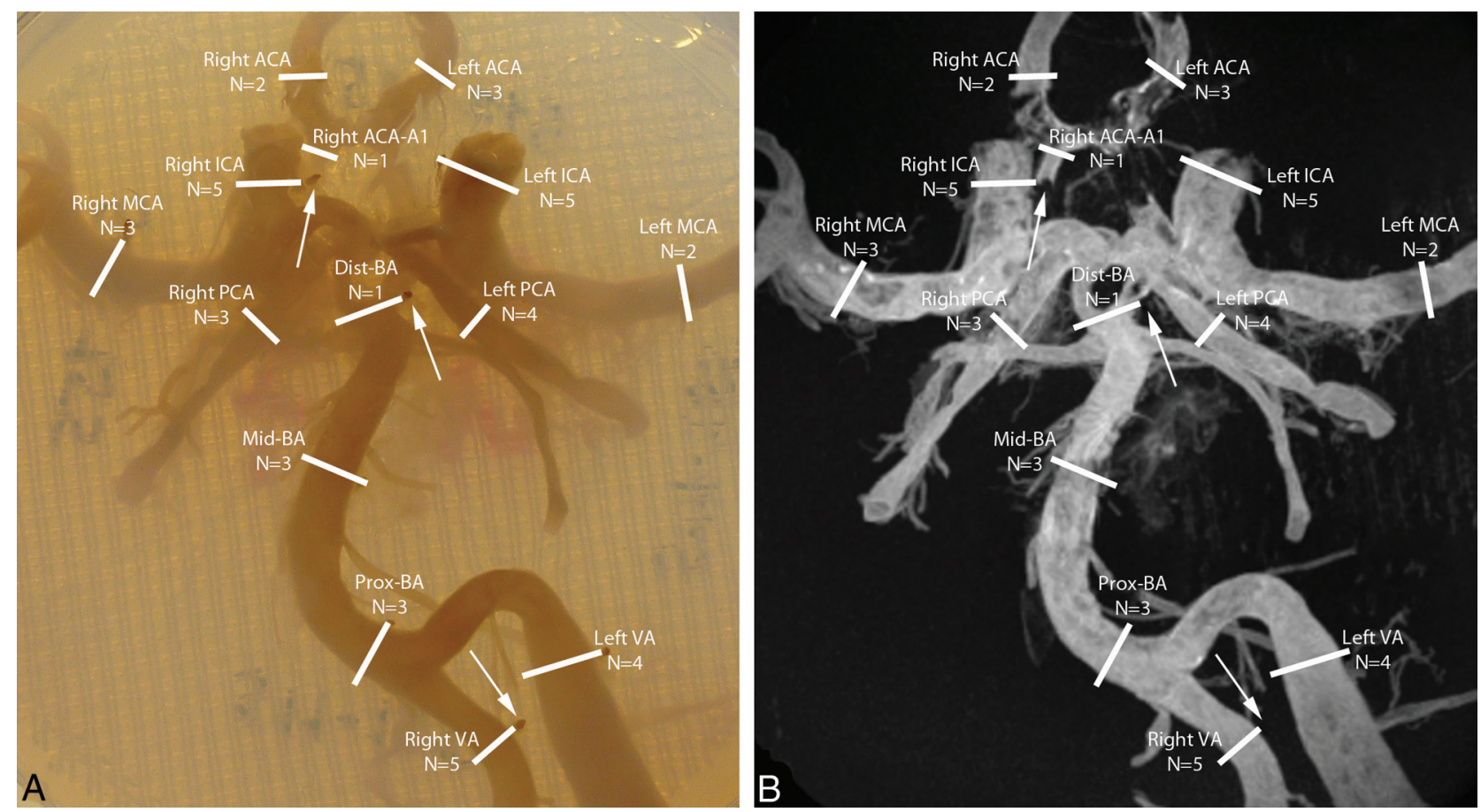

FIG 1. Photograph $(A)$ and maximum intensity projection (B) of the 7T T1-weighted turbo field echo MR imaging sequence of the CoW specimen of an 87 -year-old man embedded in a $2 \%$ agarose solution within a Petri dish. The sample locations are illustrated by white lines. For each sample location, care was taken to avoid sampling near a visible air bubble or sampling of a collapsed arterial segment. The $N$ below each arterial segment denotes the number of samples for that location within the 44 assessed samples obtained from the 5 CoW specimens. MR images were correlated with histologic sections by using fiducials placed within the agarose solution (arrows in $A$ and $B$ ). Al indicates $A 1$ segment of the anterior cerebral artery; Dist-BA, distal segment of the basilar artery; Mid-BA, middle segment of the basilar artery; Prox-BA, proximal segment of the basilar artery; ACA, anterior cerebral artery; VA, vertebral artery.

Petri dish. The tissue samples, placed within small plastic containers with 6 spaces, were then placed in the chelating reagent ethylenediaminetetraacetic acid $12.5 \%$ for 3-4 days to dissolve wall calcifications, to reduce the risk of damaging the samples during slicing. Ethylenediaminetetraacetic acid captures calcium ions within calcified areas but retains associated phosphate groups, which can afterward be visualized with an H\&E stain. After being processed and embedded in paraffin, the samples were cut into 4- $\mu \mathrm{m}$ sections, stained with H\&E and Van Gieson elastic, and assessed by using a slide scanner (Slide scanner with Dotlide software, Version 2.5; Olympus, Tokyo, Japan).

The modified American Heart Association classification by Virmani et $\mathrm{al}^{25}$ was used to classify each sample on atherosclerotic characteristics, as follows: no anomaly; early lesions, including intimal thickening ( $<50 \%$ smooth-muscle cells, no lipids, inflammatory cells), fatty streak, and pathologic intimal thickening ( $>50 \%$ smooth-muscle cells, rich in proteoglycans, foamy macrophages); and advanced lesions, including fibrolipid plaque ( $>40 \%$ lipid), thin cap atheroma $(<65-\mu \mathrm{m}$ thickness), fibrous plaque ( $<40 \%$ lipid), fibrocalcified plaque $(>40 \%$ calcified $)$, and calcified nodule (calcified element protruding into the intima). When applicable, plaque complications, like rupture, hemorrhage, or erosion, were also assessed.

\section{Correlation of MR Images with Histology}

First, T1-weighted images were reconstructed $(0.11-\mathrm{mm}$ thickness) for each sample, corresponding to the orientation and location of the PD-, T2-, and T2*-weighted images at each of the 13 locations marked by the fiducials. Then, the MR images were compared with the histologic sections at the corresponding location. If the MR images did not match the corresponding histologic section due to errors in MR imaging planning or gross deformation of the sample during histologic processing, the sample was excluded from analysis. Some deformation of the samples is inevitable; however, when no correlation in shape could be identified even when the ink locations were used as additional spatial markers, the sample was excluded. Samples were also excluded from analysis in case of histologic processing errors (mixed-up/damaged/parallel, instead of perpendicular, cut samples). The resulting MR imaging histology sets were then evaluated for agreement or disagreement between plaque components and image contrast differences. First, MR images were assessed by A.G.v.d.K. for the presence of image contrast heterogeneity within the arterial vessel wall for each sample; then, the corresponding areas on the histologic sections were assessed by N.P.D. for possible atherosclerotic changes that could explain the image contrast heterogeneity seen on the MR images.

When no vessel wall atherosclerosis was present, heterogeneities on MR imaging were scored as having "no correlation." When atherosclerosis was present in the same area, the spatial organization of plaque components (eg, collagen-rich rim, areas of foamy macrophages) was compared with the spatial organization of the vessel wall MR imaging heterogeneities, scoring either "no correlation" or a correlation that was then described more specifically. Finally, samples in which no MR imaging heteroge- 


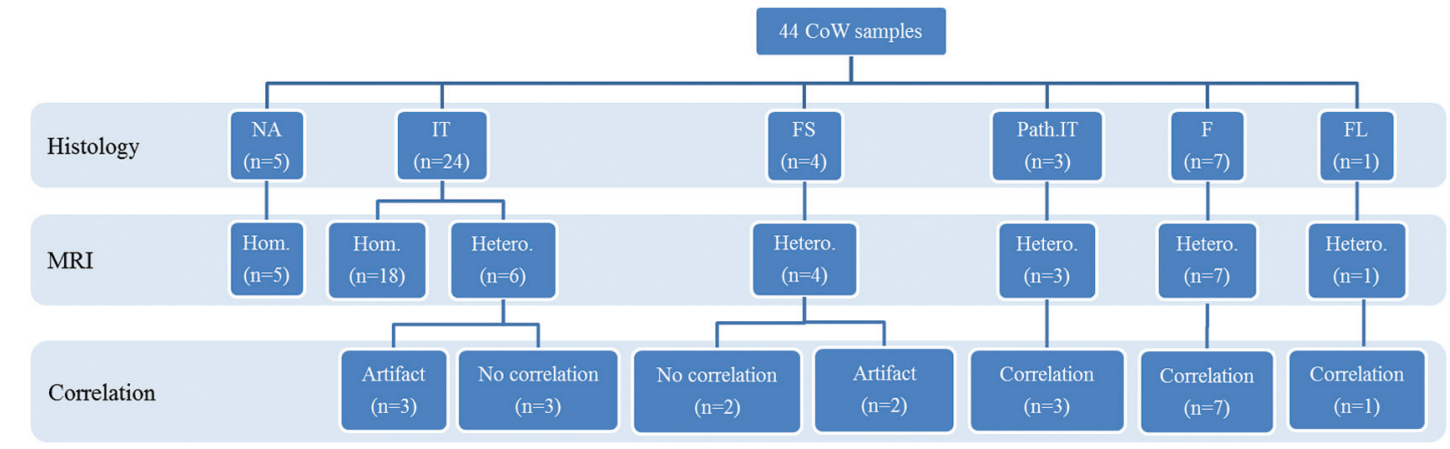

FIG 2. Overview of histologic classification and the presence of vessel wall heterogeneity on MR imaging of the 44 samples of the CoW, including correlation scoring. "Artifact" indicates intima-media artifacts; F, fibrous plaque; FL, fibrolipid plaque; FS, fatty streak; Hom., vessel wall with homogeneous signal intensity; Hetero., vessel wall with heterogeneous signal intensity; IT, intimal thickening; NA, no anomaly (no atherosclerosis); Path.IT, pathologic intimal thickening.

neity was found but where atherosclerotic changes were present were also described. Because this was a feasibility study assessing the ability of 7T MR imaging to provide sufficient image contrast for visualizing intracranial atherosclerotic plaques and characterizing its components, blinding was not performed.

\section{RESULTS}

\section{Sample Population}

Five CoW specimens from 3 men, 54 (subject 1 in figures), 71 (subject 2), and 87 years of age (subject 3), and 2 women, 65 (subject 4) and 74 years of age (subject 5), were used; although clinical information regarding disease status of these patients was not available due to the coded handling of the material, our histopathologic studies did not show characteristics of other specific diseases (vasculopathy, vasculitis) that may have adversely affected the arteries. A total of 65 samples of CoW arteries was obtained; 21 of these 65 samples (32.3\%) were excluded due to either lack of correlation in shape or fiducial location $(n=11)$, air within the sample that was not seen during MR imaging planning $(n=1)$, or histologic processing errors $(n=9)$, resulting in 44 samples for assessment. Processing samples for histologic analysis was a very delicate process, due to the fragile nature of the arterial tissue in combination with the small size of the taken samples; these features sometimes led to parallel cut samples or deformation of the sample. Figure 1 illustrates the sample location and the number of samples obtained at each location.

\section{Histologic Classification}

Five samples (11.4\%) had no atherosclerosis; 31 samples $(70.4 \%)$ contained early atherosclerotic lesions: Twenty-four samples showed intimal thickening, 4 showed a fatty streak, and 3 showed pathologic intimal thickening. The remaining 8 samples $(18.2 \%)$ showed either fibrous plaques $(n=7)$ or a fibrolipid plaque $(n=1)$. No fibrocalcific plaques, calcified nodules, or thin cap atheromas were seen; also, no plaque complications such as rupture, (intraplaque) hemorrhage, or erosion were found.

\section{MR Image Contrast Heterogeneity}

Twenty-three of 44 samples (52.3\%) showed no image-contrast heterogeneity on any of the $4 \mathrm{MR}$ image contrast weightings (T1-, PD-, T2-, or T2*-weightings; Fig 2 and On-line Figure). Samples within this group comprised solely no or early lesions: Five samples had no atherosclerosis; the other 18 showed intimal thickening (On-line Figure). In 21 of 44 samples (47.7\%), various patterns of image contrast heterogeneity were found (Fig 2 and On-line Table 2). Eleven samples (25.0\%; Fig 3) showed good correlation between the spatial organization of vessel wall MR imaging heterogeneities (areas of decreased or increased signal intensity) and the spatial organization of plaque components (eg, collagen-rich rim, areas of foamy macrophages). These 11 samples comprised 8 advanced lesions ( 7 fibrous plaques, 1 fibrolipid plaque) and 3 samples with pathologic intimal thickening (last stage of early lesions). Within these samples, areas of foamy macrophages and proteoglycans or areas with high levels of lipids were most often ( 8 of 9 plaques with these characteristics) seen as hypointense areas within the vessel wall on the PD-, T2-, and T2*weighted sequences (Fig 3); 1 sample with foamy macrophages showed a hyperintense signal on all sequences used (distal basilar artery in a 71-year-old man, On-line Table 2). Areas of increased collagen content (present in all 11 plaques) showed more ambiguous signal intensities: Five samples showed corresponding hyperintense areas on at least $\mathrm{T} 2$ - and $\mathrm{T} 2{ }^{*}$-weighted images (Fig 3, cases 1-3), with isointense-to-hyperintense signal on the T1- and $\mathrm{PD}$-weighted images, while 5 samples showed a hypointense signal for these areas on the T2- and $\mathrm{T} 2{ }^{\star}$-weighted images (Fig 3, case 4 ), with various signal intensities on the T1- and PD-weighted images. In 1 sample, the collagen-rich area could only be distinguished from healthy vessel wall because of the adjacent hypointense area of foamy macrophages.

Five other samples ( 3 with intimal thickening and 2 with fatty streaks, 11.4\%) showed image-contrast heterogeneity on MR images, without corresponding plaque components on histologic sections (Fig 4). Eleven samples (25.0\%, 5 with no other signal heterogeneity; Fig 4) showed a hypointense line within the vessel wall on the PD-, T2-, and T2*-weighted images, which was isointense on the T1-weighted images. Although this hypointensity corresponded with a space between the intima and media of the arterial wall on the histologic sections, it was regarded as artifacts of the specimen because of either detachment of the intima (eg, due to prolonged storage) or because of cleaning the specimens (flushing arteries with water/careful removal of blood clots). Due to these intima-media artifacts, making a distinction between ar- 

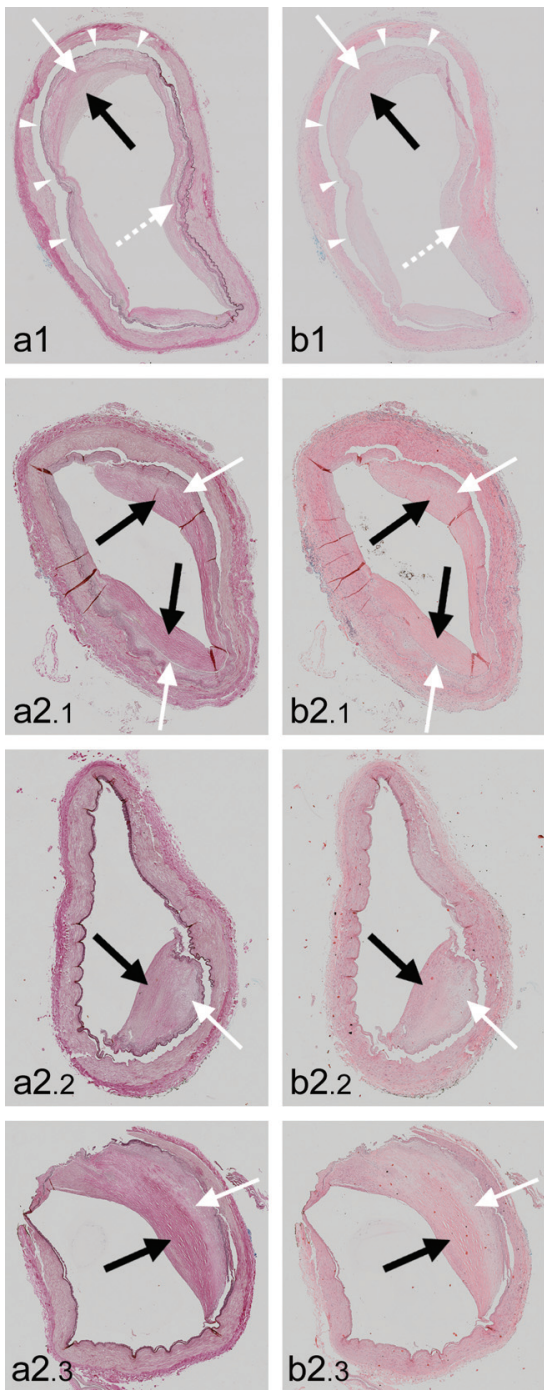
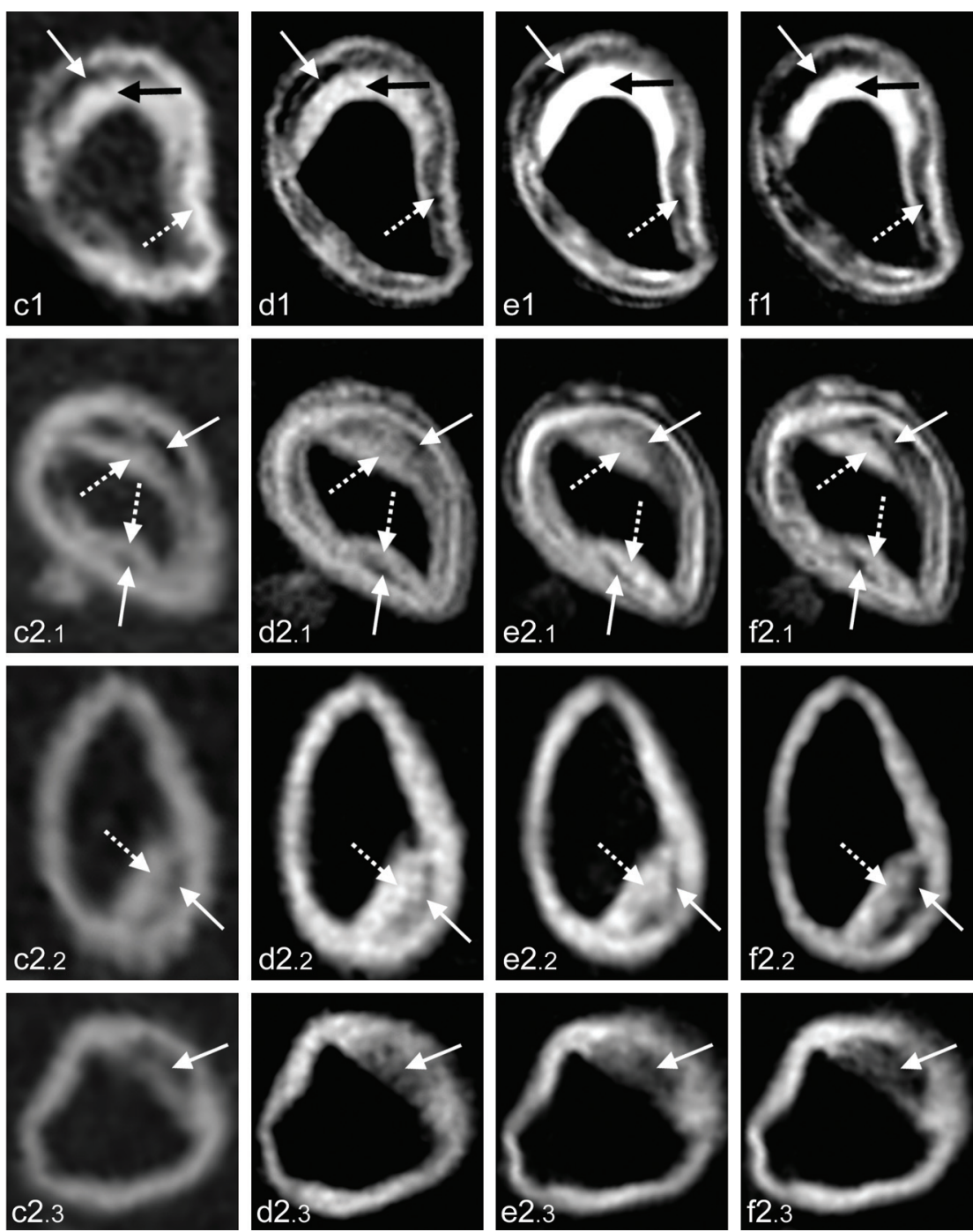

FIG 3. Four examples of atherosclerotic plaques with corresponding signal heterogeneity on 7T MR images. Histologic sections (magnification $\times 10)$ with Van Gieson elastic $(a)$ and H\&E $(b)$ staining, with corresponding 7T MR images of T1-weighted (c), PD-weighted (d), T2-weighted (e), and T2*-weighted ( $f$ ) sequences. al-fl, Cross-section of the left ICA of subject 3 . Histologic examination shows fibrous plaque with proteoglycans (white arrow, al-bl) and increased collagen (black arrow, al-bl); a patch of foamy macrophages can also be appreciated (dashed white arrow, $a l-b 7)$. On the corresponding MR images, the rim of increased collagen can be seen as hyperintense on all sequences (black arrow, cl-fl), while the small patch of foamy macrophages corresponds with a hypointense area (dashed arrow, cl-fl). Due to the intima-media artifacts, a distinction between artifacts (arrowheads, al-bl) and proteoglycans lining the artifacts within the hypointense area on MR imaging (white arrow, cl-f1) cannot be made. a2.1-f2.3, Cross-sections of the right vertebral artery (2.1), right ICA (2.2), and left ICA (2.3) of subject 2. a2.1-f2.1, Histologic examination shows fibrous plaque with increasing collagen from outside (white arrows, a2.1-b2.1) to inside (black arrows, a2.1-b2.1); on the corresponding MR images, the area with more strongly increased collagen appears as a mostly hyperintense inner area (dashed arrows, $c 2.1-f 2.7$ ), compared with the area with less collagen (white arrows, c2.1-f2.1). a2.2-f2.2, Histologic examination shows pathologic intimal thickening with proteoglycans and foamy macrophages (white arrow, a2.2-b2.2) and increased collagen (black arrow, a2.2-b2.2); again, the collagen-rich inner area appears isointense on the MR images (dashed white arrow, c2.2-f2.2), while the area with proteoglycans and foamy macrophages appears mostly hypointense (white arrow, c2.2-f2.2). a2.3-f2.3, Histologic examination shows fibrous plaque with a thick inner rim of increased collagen (black arrow, a2.3-b2.3) and a thick outer rim with foamy macrophages (white arrow, a2.3-b2.3); in this case, vessel wall thickening on MR imaging has a hypointense signal, corresponding with both foamy macrophages and increased collagen (white arrow, c2.3-f2.3).

tifacts and proteoglycans/foamy macrophages lining the artifacts within the hypointense area on MR imaging was sometimes difficult (Fig 3, case 1).

\section{DISCUSSION}

In the present study, we assessed the feasibility of 7T MR imaging to characterize ex vivo intracranial atherosclerotic plaques with sufficient image contrast, by using ultra-high-resolution sequences with 4 different contrast weightings. Areas of focal arte- rial vessel wall thickening on ultra-high-resolution MR images corresponded with histologically determined advanced atherosclerotic lesions. In all of these more advanced lesions, signal heterogeneities on 7T MR imaging enabled the spatial differentiation of different plaque components, like foamy macrophages and collagen. In early lesions, no signal-intensity heterogeneity could be observed.

Using 7T MR imaging with dedicated surface coils made it possible to image the intracranial arterial vessel wall ex vivo with 

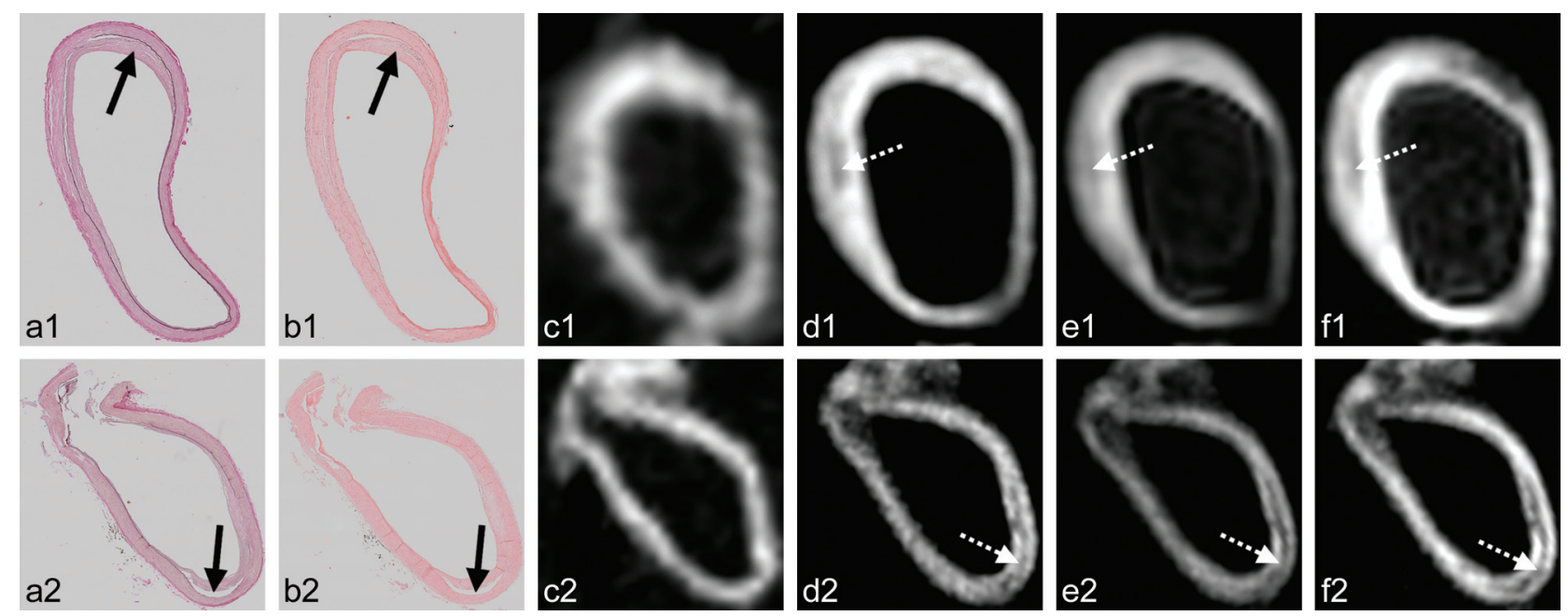

FIG 4. Two examples of discrepancies between 7T MR imaging and histology. Histologic sections (magnification $\times 10$ ) with Van Gieson elastic (a) and $\mathrm{H} \& \mathrm{E}(b)$ staining, with corresponding 7T MR images of the T1- $(c)$, PD- $(d)$, T2- $(e)$, and T2*-weighted ( $f$ ) sequences. Cross-section of the right posterior cerebral artery (al-fl) and left ICA (a2-f2) of subject 5. al-f1, Histologic examination shows a fatty streak (black arrow, al-bl), which is not seen as signal heterogeneity of the vessel wall on the MR images. A hypointense area away from the fatty streak can be appreciated on the MR images (dashed white arrow, dl-fl), but this does not correspond with any vessel wall pathology on histology, apart from intima-media artifacts. a2-f2, Histologic examination shows minor intimal thickening on the PD-, T2-, and T2*-weighted MR images. A hypointense line can be seen within a large part of the vessel wall (dashed white arrow, $d 2-f 2$ ), which corresponds to intima-media artifacts on the histologic sections (black arrow, a2-b2).

an acquired in-plane resolution of $0.11 \times 0.11 \mathrm{~mm}$. For the T1weighted turbo field echo sequence, the images could be reconstructed in all directions by using 3D image acquisitions of $0.11 \times$ $0.11 \times 0.11 \mathrm{~mm}$. With this high spatial resolution, conspicuity of the intracranial arterial vessel wall and its pathology could be obtained, though some problems of ex vivo imaging remained, such as removal of all air from the arterial lumen, that may otherwise give rise to image artifacts. An initial comparison was performed with histologic classification at specific arterial locations, similar to carotid plaque characterization studies. ${ }^{26}$ Of the 44 arterial samples that were assessed in this study, correlation between MR imaging and histology was shown to be best in the samples with more advanced lesions. None of the 5 samples with healthy arterial vessel walls showed areas of signal hypoor hyperintensity on MR images. This finding was also true for 18 of 24 samples (75\%) with intimal thickening, suggesting that these early atherosclerotic changes are beyond the contrast-to-noise ratio obtained with the ultra-high-resolution sequences used.

Of the more advanced lesions, all 8 samples ( 7 with fibrous plaque, 1 with fibrolipid plaque) showed at least partial correlation between the spatial organization of the MR signal heterogeneities and the spatial organization of plaque components of the corresponding histologic sections. In this small subset, a hypointense signal on all sequences generally corresponded to the presence of foamy macrophages, increased proteoglycans, or a lipidrich core (with or without additional intima-media artifacts). Areas of increased collagen content showed more ambiguous signal intensities ranging from hypo- to hyperintense on the same image contrast weightings. In comparison, previous studies ${ }^{27-29}$ on plaque characterization in the carotid artery showed a lipidrich core to be hyperintense on T1-weighted imaging and iso- to hypointense on PD- and T2-weighted imaging; a fibrous (collagen-rich) area was shown to be isointense on T1-weighted imag- ing, iso- to hyperintense on T2-weighted imaging, and hyperintense on PD-weighted imaging. The discrepancies in signal characteristics between these studies and our results may be due to the prolonged formalin fixation of our specimen, due to the changed contrast at ultra-high-field (where, for example, compact collagen has a shorter $\mathrm{T} 2^{* 30}$ ), or they may be related to the less advanced atherosclerotic status of most of our samples. For instance, we only had 1 sample with a lipid-rich core, and no samples with intraplaque hemorrhage or plaque rupture, advanced atherosclerotic characteristics on which the MR signal characteristics of these previous studies were mostly based. ${ }^{27-29}$ Overall, the clearest histology-corresponding image contrast heterogeneity was seen on the T2- and T2*-weighted MR images (see, for instance, Fig 3e1-f1), followed by the T1-weighted images. The $\mathrm{PD}$-weighted images showed less clear image contrast differences among different plaques components.

In $14 \%$ of the samples $(n=6)$, heterogeneity of the arterial vessel wall was found on MR imaging without histologic correlates. This could be due to the larger through-plane spatial resolution of the sequences used in this study. Although a throughplane spatial resolution of only $1 \mathrm{~mm}$ was used, the resolution obtained with histologic sections was still several factors above the MR imaging resolution. Partial volume effects within the obtained 1-mm-thick images resulted in a summation of signal intensities within this 1 -mm-thick area, while a single histologic section of $4-\mu \mathrm{m}$ thickness only showed pathology within that 4- $\mu$ m-thick area.

This study has limitations. Although CoW specimens were selected that macroscopically contained atherosclerotic plaques, only $18 \%$ of samples contained advanced plaques with corresponding plaque components. A higher percentage of advanced plaques would have given more clear insight into the specific MR signal characteristics of different plaque components (general signal intensity on different image contrast weightings), which are 
not as clearly present in early lesions. Especially, increased collagen content within atherosclerotic plaques, which showed ambiguous signal-intensity results in the current study, might benefit from inclusion of more advanced plaques. Even so, our results still show that 7T MR imaging has the image contrast to show focal thickening of the intracranial arterial vessel wall and to distinguish areas with different plaque components by using ultrahigh-resolution sequences. Furthermore, in this study, 2D sections of selective areas were used for most MR imaging; a 3D approach (like the T1-weighted sequence used) with isotropic voxels would decrease exclusion of samples due to location inconsistencies between MR imaging and histology, thereby decreasing possible selection bias. Regarding technical improvements, development of quantitative MR images, like T1-, $\mathrm{T} 2-$, and $\mathrm{T} 2{ }^{*}$ mapping, would enable quantitative plaque characterization, making qualitative scoring performed in the current study unnecessary. These improvements would not only enable more firm statements regarding the accuracy of either T1-, T2-, or T2*-weighted sequences in characterizing atherosclerotic plaque, they could also enable the development of in vivo sequences specifically designed to visualize $\geq 1$ plaque component with high image contrast, even making very high spatial resolutions unnecessary.

A hypointense line within the vessel wall was seen in $25 \%$ of samples, corresponding to a defect between the intima and media of the vessel wall. These artifacts could be related to a prolonged storage period of the CoW specimens. Although easily identifiable, it did influence our results (On-line Table 2); because the same hypointense signal is seen in foamy macrophages/lipid-rich core, we therefore could not clearly distinguish vacant space from foamy macrophages/lipid-rich core when the artifacts were present in the histologic sections. This limitation, however, will not be present with in vivo characterization of intracranial atherosclerotic plaques. Furthermore, the current ex vivo studies will be of limited use in the validation of contrast enhancement that can be visible with in vivo MR imaging. Obviously, contrast agent injection and subsequent enhancement can only be imaged in vivo, ${ }^{31}$ and postmortem examination in these patients is the only validation method. Finally, the very long scan duration (approximately 40 hours) of the ultra-high-resolution sequences used in this study prohibits their use in vivo in clinical practice. However, we think that our results may serve as a starting point for further histologic validation of in vivo intracranial vessel wall sequences, albeit with lower spatial resolutions.

\section{CONCLUSIONS}

Our results show that 7T MR imaging, by using ultra-high-resolution sequences with different image contrast weightings, has image contrast capable of identifying focal thickening of the intracranial arterial vessel walls and distinguishing areas of different signal intensities that spatially correspond to plaque components within more advanced intracranial atherosclerotic plaques. Additional studies that further validate signal characteristics of the specific plaque components in a quantitative manner, also for lower resolution sequences, will enable future in vivo characterization of intracranial atherosclerotic plaques.
Disclosures: Anja van der Kolk—RELATED: Other: Center for Translational Molecular Medicine, ${ }^{*}$ Comments: This research was performed within the framework of the Center for Translational Molecular Medicine (www.ctmm.nl), project PARISk (Plaque At RISk; grant 01C-202), and was supported by the Dutch Heart Foundation. Fredy Visser-RELATED: Employment: Philips Healthcare, Best, the Netherlands. *Money paid to the institution.

\section{REFERENCES}

1. Qureshi AI, Caplan LR. Intracranial atherosclerosis. Lancet 2014;383:984-98

2. Dieleman N, van der Kolk AG, Zwanenburg JJ, et al. Imaging intracranial vessel wall pathology using magnetic resonance imaging: current prospects and future directions. Circulation 2014;130: 192-201

3. Klein IF, Lavallee PC, Mazighi M, et al. Basilar artery atherosclerotic plaques in paramedian and lacunar pontine infarctions: a high-resolution MRI study. Stroke 2010;41:1405-09

4. Xu WH, Li ML, Gao S, et al. Plaque distribution of stenotic middle cerebral artery and its clinical relevance. Stroke 2011;42: 2957-59

5. van der Kolk AG, Zwanenburg JJ, Brundel M, et al. Intracranial vessel wall imaging at 7.0-T MRI. Stroke 2011;42:2478-84

6. Skarpathiotakis M, Mandell DM, Swartz RH, et al. Intracranial atherosclerotic plaque enhancement in patients with ischemic stroke. AJNR Am J Neuroradiol 2013;34:299-304

7. Lou X, Ma N, Ma L, et al. Contrast-enhanced 3T high-resolution MR imaging in symptomatic atherosclerotic basilar artery stenosis. AJNR Am J Neuroradiol 2013;34:513-17

8. van der Kolk AG, Hendrikse J, Brundel M, et al. Multi-sequence whole-brain intracranial vessel wall imaging at 7.0 Tesla. Eur Radiol 2013;23:2996-3004

9. Turan TN, Bonilha L, Morgan PS, et al. Intraplaque hemorrhage in symptomatic intracranial atherosclerotic disease. J Neuroimaging 2011;21:e159-61

10. Park JK, Kim SH, Kim BS, et al. Imaging of intracranial plaques with black-blood double inversion recovery MR imaging and CT. J Neuroimaging 2011;21:e64-68

11. Chung JW, Kim BJ, Choi BS, et al. High-resolution magnetic resonance imaging reveals hidden etiologies of symptomatic vertebral arterial lesions. J Stroke Cerebrovasc Dis 2014;23: 293-302

12. Natori T, Sasaki M, Miyoshi M, et al. Evaluating middle cerebral artery atherosclerotic lesions in acute ischemic stroke using magnetic resonance T1-weighted 3-dimensional vessel wall imaging. $J$ Stroke Cerebrovasc Dis 2014;23:706-11

13. Turan TN, Rumboldt Z, Brown TR. High-resolution MRI of basilar atherosclerosis: three-dimensional acquisition and FLAIR sequences. Brain Behav 2013;3:1-3

14. Yuan C, Mitsumori LM, Ferguson MS, et al. In vivo accuracy of multispectral magnetic resonance imaging for identifying lipid-rich necrotic cores and intraplaque hemorrhage in advanced human carotid plaques. Circulation 2001;104:2051-56

15. Cai JM, Hatsukami TS, Ferguson MS, et al. Classification of human carotid atherosclerotic lesions with in vivo multicontrast magnetic resonance imaging. Circulation 2002;106:1368-73

16. Moody AR, Murphy RE, Morgan PS, et al. Characterization of complicated carotid plaque with magnetic resonance direct thrombus imaging in patients with cerebral ischemia. Circulation 2003;107:3047-52

17. Mitsumori LM, Hatsukami TS, Ferguson MS, et al. In vivo accuracy of multisequence MR imaging for identifying unstable fibrous caps in advanced human carotid plaques. J Magn Reson Imaging 2003; $17: 410-20$

18. Cappendijk VC, Cleutjens KB, Kessels AG, et al. Assessment of human atherosclerotic carotid plaque components with multisequence MR imaging: initial experience. Radiology 2005;234:487-92

19. Narumi S, Sasaki M, Ohba H, et al. Predicting carotid plaque char- 
acteristics using quantitative color-coded T1-weighted MR plaque imaging: correlation with carotid endarterectomy specimens. AJNR Am J Neuroradiol 2014;35:766-71

20. den Hartog AG, Bovens SM, Koning W, et al. Current status of clinical magnetic resonance imaging for plaque characterisation in patients with carotid artery stenosis. Eur J Vasc Endovasc Surg 2013;45:7-21

21. Majidi S, Sein J, Watanabe M, et al. Intracranial-derived atherosclerosis assessment: an in vitro comparison between virtual histology by intravascular ultrasonography, 7T MRI, and histopathologic findings. AJNR Am J Neuroradiol 2013;34:2259-64

22. Jahnke C, Dietrich T, Paetsch I, et al. Experimental evaluation of the detectability of submillimeter atherosclerotic lesions in ex vivo human iliac arteries with ultrahigh-field (7.0 T) magnetic resonance imaging. Int J Cardiovasc Imaging 2007;23:519-27

23. Petridou N, Italiaander M, van de Bank BL, et al. Pushing the limits of high-resolution functional MRI using a simple high-density multi-element coil design. NMR Biomed 2013;26:65-73

24. Versluis MJ, Peeters JM, van RS, et al. Origin and reduction of motion and $\mathrm{f} 0$ artifacts in high resolution $\mathrm{T} 2^{\star}$-weighted magnetic resonance imaging: application in Alzheimer's disease patients. $\mathrm{Neu}$ roimage 2010;51:1082-88

25. Virmani R, Kolodgie FD, Burke AP, et al. Lessons from sudden cor- onary death: a comprehensive morphological classification scheme for atherosclerotic lesions. Arterioscler Thromb Vasc Biol 2000;20: 1262-75

26. Saam T, Hatsukami TS, Takaya N, et al. The vulnerable, or high-risk, atherosclerotic plaque: noninvasive MR imaging for characterization and assessment. Radiology 2007;244:64-77

27. Yuan C, Mitsumori LM, Beach KW, et al. Carotid atherosclerotic plaque: noninvasive MR characterization and identification of vulnerable lesions. Radiology 2001;221:285-99

28. Fuster V, Fayad ZA, Moreno PR, et al. Atherothrombosis and highrisk plaque: Part II: approaches by noninvasive computed tomographic/magnetic resonance imaging. J Am Coll Cardiol 2005;46: 1209-18

29. Silvera SS, Aidi HE, Rudd JH, et al. Multimodality imaging of atherosclerotic plaque activity and composition using FDG-PET/CT and MRI in carotid and femoral arteries. Atherosclerosis 2009;207: $139-43$

30. de Jong S, Zwanenburg JJ, Visser F, et al. Direct detection of myocardial fibrosis by MRI. J Mol Cell Cardiol 2011;51:974-79

31. Portanova A, Hakakian N, Mikulis DJ, et al. Intracranial vasa vasorum: insights and implications for imaging. Radiology 2013; 267:667-79 